\title{
Abscisic Acid Mediates Drought-Enhanced Rhizosheath Formation in Tomato
}

\author{
Joseph K. Karanja ${ }^{1}$, Mehtab Muhammad Aslam ${ }^{1}$, Zhang Qian ${ }^{1}$, Richard Yankey ${ }^{2}$, \\ Ian C. Dodd ${ }^{3}$ and Xu Weifeng ${ }^{1 *}$ \\ ${ }^{1}$ Center for Plant Water-Use and Nutrition Regulation and College of Life Sciences, Joint International Research Laboratory \\ of Water and Nutrient in Cops, Fujian Agriculture and Forestry University, Fuzhou, China, ${ }^{2}$ China National Engineering \\ Research Center of Juncao Technology, Fujian Agriculture and Forestry University, Fuzhou, China, ${ }^{3}$ The Lancaster \\ Environment Centre, Lancaster University, Lancaster, United Kingdom
}

OPEN ACCESS

Edited by:

Vicent Arbona,

University of Jaume I, Spain

Reviewed by:

Dong-Ha Oh,

Louisiana State University,

United States

Min Zhu,

Yangzhou University, China

*Correspondence: Xu Weifeng

wfxu@fafu.edu.cn

Specialty section:

This article was submitted to

Plant Abiotic Stress,

a section of the journal

Frontiers in Plant Science

Received: 26 January 2021

Accepted: 23 June 2021

Published: 23 July 2021

Citation:

Karanja JK, Aslam MM, Qian Z,

Yankey $R$, Dodd IC and Weifeng $X$

(2021) Abscisic Acid Mediates

Drought-Enhanced Rhizosheath

Formation in Tomato.

Front. Plant Sci. 12:658787.

doi: 10.3389/fpls.2021.658787
The rhizosheath, commonly defined as soil adhering to the root surface, may confer drought tolerance in various crop species by enhancing access to water and nutrients under drying stress conditions. Since the role of phytohormones in establishing this trait remains largely unexplored, we investigated the role of $A B A$ in rhizosheath formation of wild-type (WT) and ABA-deficient (notabilis, not) tomatoes. Both genotypes had similar rhizosheath weight, root length, and root ABA concentration in well-watered soil. Drying stress treatment decreased root length similarly in both genotypes, but substantially increased root ABA concentration and rhizosheath weight of WT plants, indicating an important role for $\mathrm{ABA}$ in rhizosheath formation. Neither genotype nor drying stress treatment affected root hair length, but drying stress treatment decreased root hair density of not. Under drying stress conditions, root hair length was positively correlated with rhizosheath weight in both genotypes, while root hair density was positively correlated with rhizosheath weight in well-watered not plants. Root transcriptome analysis revealed that drought stress increased the expression of ABA-responsive transcription factors, such as AP2-like ER TF, alongside other drought-regulatory genes associated with $\mathrm{ABA}$ (ABA 8'-hydroxylase and protein phosphatase $2 \mathrm{C}$ ). Thus, root ABA status modulated the expression of specific gene expression pathways. Taken together, drought-induced rhizosheath enhancement was ABA-dependent, but independent of root hair length.

Keywords: ABA, drought stress, transcriptome analysis, rhizosheath formation, tomato

\section{INTRODUCTION}

Drought conditions often limit plant growth and yields in agricultural systems (Mahalingam, 2015; Anjum et al., 2017; Hussain et al., 2018), thereby threatening the food security of the ever-surging global population (Long et al., 2015). The frequency and severity of drought are predicted to increase with climate change (Battisti and Naylor, 2009; Mach et al., 2019). Drying stress modifies root system architecture (RSA) to increase water uptake, potentially enhancing plant growth and yields (Jeong et al., 2013; Uga et al., 2013). RSA describes the three-dimensional organization of different root types (e.g., primary and laterals) in the soil (Lynch, 1995; Ning et al., 2012; Smith and De Smet, 2012). Deeper roots and increased root density can enhance plant growth under 
water deficit conditions by enhancing water and nutrient acquisition from the heterogeneous soil environment (Lynch et al., 2014; Zhan et al., 2015). Plant hormones play a crucial role in root growth and development (Davies, 2010). Among classical phytohormones, abscisic acid (ABA) has been widely considered as a stress hormone, and its role in regulating plant drought stress responses has been extensively studied (Schachtman and Goodger, 2008; Cutler et al., 2010). Drying stress treatment stimulates ABA accumulation, which plays an important role in maintaining root elongation (Saab et al., 1990; Giuliani et al., 2005) as well as root hair formation and elongation in Arabidopsis and crop plants (Chen et al., 2006; Xu et al., 2013).

At a microscopic scale, root architecture includes root hairs, tubular extensions of root epidermal cells that emerge behind the root elongation zone (Peterson and Farquhar, 1996; Richardson et al., 2009; Pereg and McMillan, 2015). Root hairs account for $70-90 \%$ of the total root surface area, and increase the soil volume from which roots can acquire resources (Smith and De Smet, 2012; Kwasniewski et al., 2016). Enhanced root hair formation is one of many potential mechanisms by which plants can enhance tolerance to soil water deficits (White et al., 2013a,b). Although WT and root hairless mutants had similar physiological and agronomic responses when grown with adequate water availability, WT plants had better water status and lower foliar ABA concentration under soil water deficit conditions (Marin et al., 2020), indicating the adaptive significance of root hairs.

The rhizosheath is defined as soil adhering to roots upon excavation, and it may enhance water status of root tissues as the soil dries (George et al., 2014). Rhizosheath formation is influenced by several factors, such as root and/or microbial mucilage (Watt et al., 1994; McCully, 1999; Carminati et al., 2017), microbial activities (Hanna et al., 2013), soil physicochemical properties (Haling et al., 2014), and root hair traits (Haling et al., 2010). Long root hairs have been associated with larger rhizosheaths in barley and wheat genotypes (Haling et al., 2014; Delhaize et al., 2015), but few studies have described the genetics of rhizosheath formation under water deficit conditions (George et al., 2014), or determined the involvement of plant hormones in rhizosheath formation.

Most attention has focused on the role of ABA in mediating rhizosheath formation by affecting root hair elongation. While $1 \mu \mathrm{M}$ exogenous ABA inhibits Arabidopsis root hair growth in vitro through transcriptional regulation (Rymen et al., 2017), similar ABA concentrations stimulated root hair elongation of hydroponically grown rice seedlings (Wang et al., 2017). While attenuating ABA signaling by overexpressing OsABIL2 in rice produced shorter root hairs (Wang et al., 2017), the root hair phenotype of ABA-deficient mutants has attracted little attention. When grown hydroponically, the ABA deficient Az34 barley mutant had a shorter root hair zone than WT plants (Sharipova et al., 2016), which might explain its limited rhizosheath formation independent of soil water availability (Zhang et al., 2021). Conversely, barley genotypes that either had or lacked root hairs (and thus differed in rhizosheath formation (George et al., 2014) had similar root xylem ABA concentrations when young vegetative plants were grown under drying stress conditions (Dodd and Diatloff, 2016). However, the involvement of $\mathrm{ABA}$ in rhizosheath formation remains obscure, especially for dicotyledonous crops such as tomato.

Other studies have identified some potential genes (e.g., glutamate receptor GLR3.1) that may explain the genetics of barley rhizosheath (George et al., 2014) possibly by enhancing root growth (Li et al., 2006; Gamuyao et al., 2012). Many genes encoding transcription factors (TFs), either induced by ABA treatment or ABA independent, have been identified in roots responsive to drying stress (Recchia et al., 2013; Janiak et al., 2016). For example, a gene encoding dehydrationresponsive element-binding1 (DREB 1) belonging to the wider family APETALA2 ethylene-responsive element-binding TFs was upregulated in maize roots under water stress conditions (Liu et al., 2013). Hence, water deficit stress perception, signaling, and regulatory pathways controlling the expression of stressresponsive genes mediate root growth, which may increase soil moisture capture via enhanced root-soil contact to mitigate the effects of drought.

Therefore, this study aimed to investigate morphological (root hair traits) and gene expression mechanisms regulating rhizosheath development in tomato, and the role of ABA in this process. We hypothesized that WT tomato plants will form more rhizosheath under drying stress conditions, relative to an ABAdeficient mutant, because of a root hair phenotype that better allowed sand binding.

\section{MATERIALS AND METHODS}

\section{Plant Material and Growth Conditions}

Seeds of tomato (Solanum lycopersicum L. cv. Lukullus) and an ABA-deficient mutant (notabilis-not) were obtained from Tomato Genetics Resource Center (University of California, Davis). The not mutant has a defect in the gene LeNCED1, which encodes a 9-cis-epoxycarotenoid dioxygenase involved in xanthoxin synthesis, a key step in ABA biosynthesis (Thompson et al., 2004).

The seeds were sterilized in a $2.6 \%$ sodium hypochlorite solution for $30 \mathrm{~min}$, and then rinsed for $1 \mathrm{~h}$ in flowing tap water. The seeds were then sandwiched between two wet Whatman No. 2 filter papers in Petri dishes and placed in the dark to germinate for 5 days. Five-day-old seedlings were transplanted into cylindrical plastic pots $(13.5-\mathrm{cm}$ inner diameter, $16-\mathrm{cm}$ height) filled with sieved sand ( $\varnothing \leq 0.85 \mathrm{~mm}$ ), which was collected from Fujian Agricultural and Forestry University botanical beach garden, and watered to different levels: well-watered (WW, 14\%), drying stress (DS, 5\%); \% shows the water content relative to the weight of sand per pot. Pots were covered with black plastic paper material to protect roots from light. They were then transferred to a greenhouse where growth conditions were set at $25 \pm 2{ }^{\circ} \mathrm{C}$, $60 \%$ relative humidity, and light intensity of $150 \mu \mathrm{mol} \mathrm{m}^{-2} \mathrm{~s}^{-1}$ (Humbeck et al., 1994), supplied by fluorescent lamps fitted with a timer set at $16 \mathrm{~h} / 8 \mathrm{~h}$ light/dark photoperiod. All the pots were watered daily to their respective irrigation regimes for 30 days. Three replicates, with two seedlings per pot, were used for each treatment. Five plants with uniform growth were selected for root traits analysis. 


\section{Rhizosheath Quantification}

After 30 days of growth, the pots were carefully disassembled, and the root columns were carefully collected and shaken to remove sand not adhered to the root surface, while minimizing disturbance to retain root-sand contact. The roots were detached from the shoots and weighed along with rhizosheath sand. roots were washed in a small jar, and the resulting rhizosheath sand and water in the small jar was dried in a tray at $105^{\circ} \mathrm{C}$ for 3 days to determine the rhizosheath dry weight. Total root length was determined using an Epson scanner (Epson, Herts, United Kingdom), and the WinRHIZO software (Regent Instruments, Quebec, Canada). The resulting rhizosheath sand and water in the small jar were dried in a tray at $105^{\circ} \mathrm{C}$ for 3 days to determine the rhizosheath dry weight. Rhizosheath weight per root length was obtained by dividing the total rhizosheath of the individual plant by its corresponding total root length.

\section{Root Hair Traits Analysis}

Three root apical segments $(1 \mathrm{~mm})$ excised from each of the 5 experimental plants per genotype/treatment combination were viewed under a Leica stereomicroscope (MZ10F, Germany). Images (JPG format) were captured using a DS-U3 camera (Nikon, Tokyo, Japan) with appropriate magnification $(40 \times)$. The length of a randomly selected root hair from each root apical segment was determined using the Image J software (National Institutes of Health; Supplementary Figure 1). Root hair density was determined as the number of root hairs in each root segments, as previously described (Nestler et al., 2016). The root hair length and density of each individual plant were calculated as the average of the three measurements.

\section{Root ABA Concentration}

In an independent experiment, WT and not seedlings were grown under the conditions described above. After $\sim 4$ weeks of growth, a root segment ( $\sim 200 \mathrm{mg}$ root dry weight) was excised, briefly washed to remove adhering sand particles, frozen in liquid nitrogen, freeze dried, and then finely ground in a bead beater (Qiagen, Hilden, Germany) with 3-mm beads at $25 \mathrm{~Hz} / \mathrm{s}$ for $3 \mathrm{~min}$. Briefly, $200 \mathrm{mg}$ of the sample was placed in a $2-\mathrm{ml}$ tube, then $400 \mu$ l ethyl acetate was added, and the mixture was homogenized. Homogenates were centrifuged at $13,000 \times \mathrm{g}$ for $10 \mathrm{~min}$ at $4^{\circ} \mathrm{C}$. Supernatant was transferred into a 2 -ml tube. After the second addition of $0.5 \mathrm{ml}$ ethyl acetate with added internal standards ( $15 \mathrm{ng}$ of ${ }^{2} \mathrm{H}_{6}$-ABA) as described by McAdam (2015), the extracts were vacuum-dried at $30^{\circ} \mathrm{C}$. The extracts were then dissolved in $70 \%$ methanol, vortexed for $20 \mathrm{~min}$, and again centrifuged at $13,000 \times \mathrm{g}$ for $10 \mathrm{~min}$ at $4^{\circ} \mathrm{C}$. The supernatant was carefully transferred to $1.5-\mathrm{ml}$ vials and then injected into the liquid chromatography system.

The samples were analyzed by HPLC-electrospray ionization/MS using Agilent 100 HPLC (Agilent, Santa Clara, CA, United States) coupled with Applied Biosystems Q-TRAP 2000 (Applied Biosystems, California, Foster City, United States). Chromatographic separation was carried out on a $3 \mu \mathrm{m} \mathrm{C18}$ $100 \times 2 \mathrm{~mm}$ column at $35^{\circ} \mathrm{C}$. The mobile phases consisted of solvents A and B (containing $0.1 \%$ formic acid and acetonitrile, respectively). Solvent gradient elution mode was programmed as follows: $5-60 \%$ B for $0-7.5 \mathrm{~min}$ and $60-95 \%$ B for $7.5-10 \mathrm{~min}$. The column was then washed with $95 \%$ B for 3 min and finally equilibrated with $100 \% \mathrm{~A}$ for $10 \mathrm{~min}$. The injection and flow rates were $2 \mu \mathrm{l}$ and. $4 \mathrm{ml} / \mathrm{min}$, respectively. MS analysis was performed by negative ion mode electrospray ionization (ESI). Optimal conditions were set using the Quantitative Optimization feature (Analyst software) by infusing MS standards with syringe pump while injecting standards into a $200 \mu \mathrm{l} / \mathrm{min}$ flow of $50 \%$ solvent $\mathrm{A} / 50 \%$ solvent $\mathrm{B}$, and were as follows: cone voltage, $40 \mathrm{~V}$; capillary voltage, $3 \mathrm{kV}$; temperature, $400^{\circ} \mathrm{C}$; desolvation gas flow, $900 \mathrm{~L} / \mathrm{h}$; cone gas flow, $50 \mathrm{~L} / \mathrm{h}$.

\section{RNA Extraction}

In an independent experiment, WT and not seedlings were grown under the conditions described above. Three biological replicates, each containing two plants of each treatment were used. At 4 weeks of growth, about $200 \mathrm{mg}$ root weight of WW and DS plants was harvested, cleaned carefully, immediately frozen in liquid nitrogen, and then stored at $-80^{\circ} \mathrm{C}$ for further analysis. Total RNA was extracted using the TRIzol ${ }^{\circledR}$ kit (Invitrogen, Carlsbad, CA, United States), following the instructions of the manufacturer. Isolated RNA was dissolved in nuclease-free water, and its quality and quantity were estimated by the Agilent Bioanalyzer 2100 system (Agilent Technologies, Santa Clara, CA, United States).

\section{Illumina RNA Sequencing and Analysis}

Equal amounts of RNA samples from WW and DS roots were prepared for RNA-sequencing (RNA-Seq). Sequencing libraries were constructed using NEBNext ${ }^{\circledR}$ Ultra $^{\text {TM }}$ RNA Library Prep Kit for Illumina (NEB, Ipswich, MA, USA), following the instructions of the manufacturer. The libraries were sequenced using the BGISEQ-500 sequencer (Beijing Genomics Institute; BGI, Shenzhen, China: Accession no. PRJNA731295). Raw reads obtained from the RNA-Seq were cleaned using SOAPnuk (version 1.5.2). Low-quality reads and those containing adapters or poly-N were eliminated. The resulting high-quality reads were mapped against the $S$. lycopersicum reference genome (ITAG4.0) using HISAT2 (version 2.2.5).

\section{Differential Gene Expression Analysis and Functional Annotation}

To estimate abundance and align reads, a method based on RNA-Seq by Expectation Maximization (RSEM) (Li and Dewey, 2011) was adopted, and bowtie2 (version 2.2.5) was chosen as the alignment method (Mascher et al., 2017). The RSEM method was used to generate expression value matrices, which were normalized as read per million per kilo base (RPKM) by dividing raw reads number multiplied by 1 billion for the transcript length multiplied by total number of mapped reads on each library.

DESeq2 (http://www.biocunductor.org/packages/release/ bioc/html/DESeq2.html) was used for differential expression analysis of the four groups (three biological replications per group). The differential expression of transcripts was tested by their significance performing the Fisher's exact test with a $p$-value cutoff $\leq 0.001$. The resulting $P$-values were adjusted using the Benjamin and Hochberg approach for dealing with the false 
discovery rate (FDR) (Benjamini and Hochberg, 1995). The false discovery rate-adjusted $q$-values were calculated using the Benjamini and Hochberg procedure. The $\log _{2}$ (fold change) for each gene was calculated. Genes with an adjusted $p$-value $\leq 0.001$ $\left[\mid \log _{2}\right.$ (fold change $\left.\mid\right)>1$ ] found by DESeq 2 were considered as differentially expressed genes (DEGs).

To assign gene annotations and gene ontology (GO) terms to the predicted tomato genes, a platform based on ITAG4.0 and GO (http://www.geneontology.org/) was used. DEGs were subjected to GO enrichment analysis. The $P$-values from the Fisher's exact test were adjusted using the Benjamini and Hochberg's approach to control the FDR. The GO terms with FDR $<0.001$ were considered significantly enriched within the gene set.

\section{KEGG Enrichment Analysis}

Kyoto Encyclopedia of Genes and Genomes is a knowledge database for systematic analysis of gene functions and linking genomic information generated by genome sequencing with higher order functional information. The Kyoto Encyclopedia of Genes and Genomes (KEGG) Orthology Based Annotation System (KOBAS, version 2.0) software was used to analyze statistical enrichment of differentially expressed transcripts in KEGG pathways (http://kobas.cbi.pku.edu.cn/; Xie et al., 2011).

\section{RT-qPCR Validation}

To validate the expression profiles of DEGs obtained from the RNA-Seq analysis, six pairs of primers (Supplementary Table 1) were designed using Primer Express Software (Applied Biosystems, Waltham, MA, United States). To perform RTqPCR, isolated RNA was reverse transcribed into cDNA using PrimeScript RT Reagent Kit with gDNA Eraser (Takara, Dalian, China), following the instructions of the manufacturer. Each reaction contained a total volume of $10 \mu \mathrm{l}$ with $1 \mu \mathrm{l}$ of diluted first strand CDNA, $5 \mu$ l of SYBR Green PCR Master Mix (Applied Biosystems, Waltham, MA, United States), and $10 \mu \mathrm{M}$ of forward and reverse primer. Reactions were performed in Applied Biosystems 7500 Real-Time PCR Systems. The ubiquitin gene was used as an internal control to normalize the expression levels (Czechowski et al., 2005). For each treatment, reactions were performed with three biological replicates pooled from three technical replicates. RT-qPCR results were analyzed using the $2^{-\Delta \Delta \mathrm{Ct}}$ method (Livak and Schmittgen, 2001).

\section{Statistical Analysis}

Seven independent experiments of the same experimental design were performed, each comprising five replicates of each genotype $x$ treatment combination. Rhizosheath and root traits were measured in experiments 1 to 5 , root ABA concentration in experiment 6 , and RNA-sequencing in experiment 7 . Root traits (rhizosheath weight, root length and their ratio, root hair length, and density) and root $\mathrm{ABA}$ concentration were analyzed by two-way ANOVA at $p \leq 0.05$ significance level using SPSS (version 1.70). Tukey's test was performed for post hoc multiple comparisons within groups. Normality of data was evaluated using homogeneity of variance test. Correlation analysis was performed using Spearman's rank correlation tests $\left(p \leq 0.05 ; r^{2}\right.$ reported $=$ rho squared), with analysis of covariance performed to determine whether different root traits affected rhizosheath weight differently in the two genotypes. DEGs analysis was performed using DEGseq (version 1.18.0) in Bioconductor package; where genes with 2 -fold difference and an adjusted $P$ value of $\leq 0.001$ were statistically significant. For RT-qPCR data analysis, Student's $t$-test $(p \leq 0.05)$ in SPSS was applied.

\section{RESULTS}

\section{Root ABA Concentration Mediates Rhizosheath Size in Drying Sand}

While there was no significant genotypic difference in root length under well-watered (WW) conditions, roots of not plants were $23 \%$ longer than those of WT plants when grown under drying stress conditions (Figure 1A). Although absolute rhizosheath mass was similar in both genotypes under WW conditions, the rhizosheath mass of WT and not root systems increased by 1.8- and 1.2-fold, respectively as the sand dried (Figure 1B). When the rhizosheath mass was normalized by root length (the specific rhizosheath mass), there were no significant genotypic differences in the WW plants, but drying stress increased specific rhizosheath mass by 3.1- and 1.9-fold in WT and not, respectively (Figure 1C). While root ABA concentrations were similar under WW conditions, drying stress treatment increased root the ABA concentration of WT plants by 2.9-fold but had no effect on not (Figure 1F). Thus, ABA accumulation was required for maximal rhizosheath development as the sand dried.

Drying stress also modified root hair traits. WT plants tended $(P=0.052)$ to have longer root hairs, while drying stress decreased root hair length similarly by almost $16 \%$ in both genotypes (Figure 1D), as indicated by the no significant genotype $\times$ watering treatment interaction (Table 1 ). In contrast, the root hair density of the WT plants was significantly $(p<$ 0.0001) greater than that of not (Figure 1E), with the root hair density of both genotypes decreasing similarly by $20 \%$ as the sand dried. Thus, drying stress decreased root hair length and root hair density independently of root ABA accumulation.

Under well-watered conditions, rhizosheath weight tended to increase $(P=0.055)$ with root length in both genotypes (Figure 2A). Under drying stress conditions, rhizosheath weight significantly increased with root length in the not plants, but rhizosheath weight was independent of root length in the WT plants. While there was no relationship between rhizosheath weight and root hair length under WW conditions, rhizosheath weight significantly increased with root hair length under drying stress conditions in both genotypes (Figure 2B). In dry sand, the WT plants bound 1.5-fold more sand per unit of root hair length. Generally, rhizosheath weight was not correlated with root hair density, but it significantly increased with root hair density in WW not plants (Figure 2C). Taken together, root hair length influenced rhizosheath weight more than root hair density when plants were grown in dry sand. 


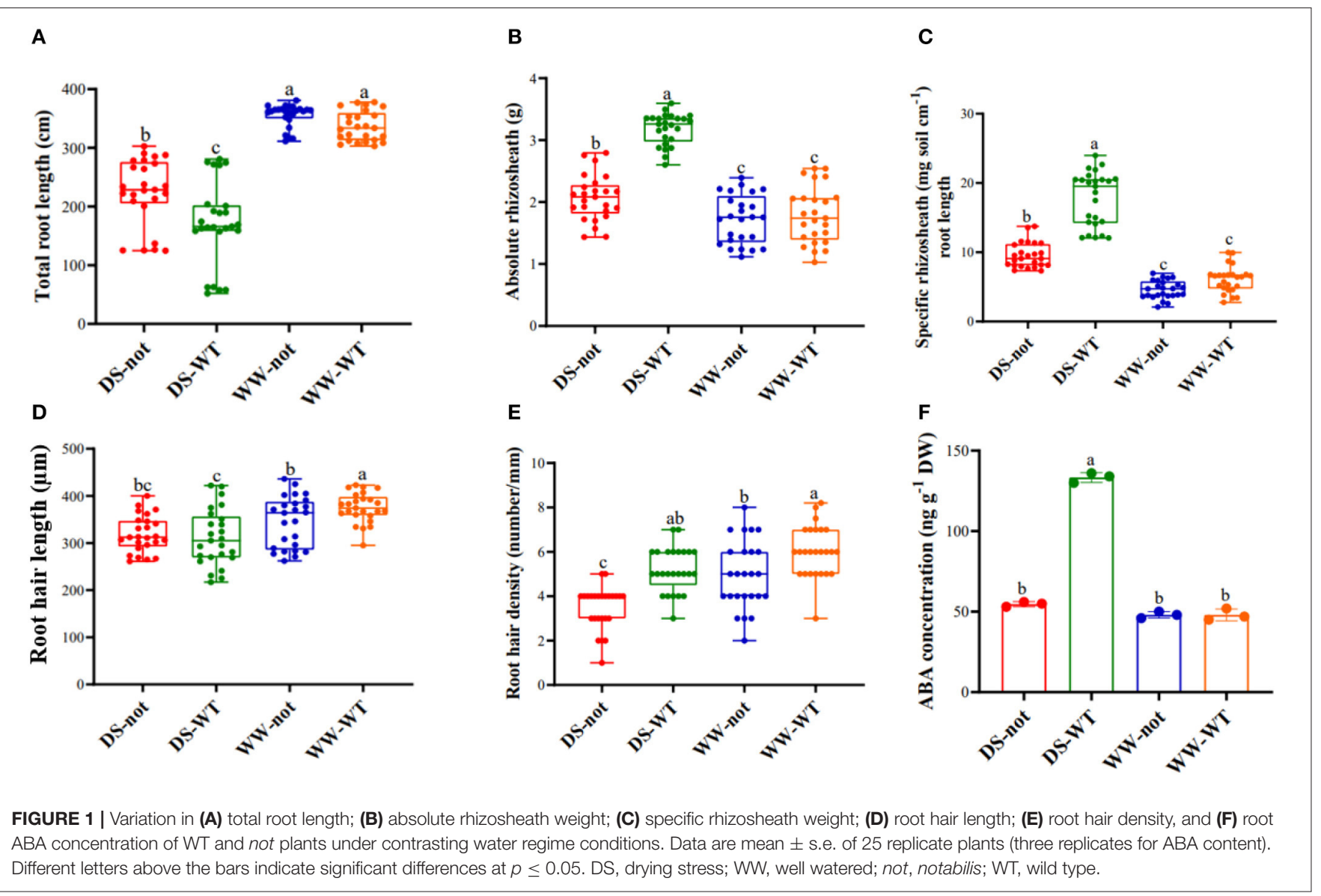

TABLE 1 | Root length, rhizosheath, root hair length and density, and root ABA concentration of not and WT plants grown under contrasting water regime conditions, with $P$-values for genotype $(G)$, watering treatment $(W)$, and their interaction $(G \times W)$ indicated.

\begin{tabular}{|c|c|c|c|}
\hline & \multicolumn{3}{|c|}{$P$-value } \\
\hline & Genotype & Watering & $\mathbf{G} \times \mathbf{W}$ \\
\hline Total root length (cm) & $<0.0001$ & $<0.0001$ & 0.074 \\
\hline Absolute rhizosheath (g) & $<0.0001$ & $<0.0001$ & $<0.0001$ \\
\hline Specific rhizosheath $\left(\mathrm{mg} \mathrm{cm}^{-1}\right)$ & 0.002 & $<0.0001$ & 0.005 \\
\hline Root hair length ( $\mu \mathrm{m})$ & 0.052 & $<0.0001$ & 0.23 \\
\hline Root hair density (number $\mathrm{mm}^{-1}$ ) & $<0.0001$ & $<0.0001$ & 0.071 \\
\hline ABA (ng g $\left.g^{-1} \mathrm{DW}\right)$ & $<0.0001$ & $<0.0001$ & $<0.0001$ \\
\hline
\end{tabular}

\section{RNA-Seq Global Analysis}

The 12 libraries were sequenced using the Illumina sequencing platform and 48,120,000-51,090,000 raw reads were obtained. An average of $6.78 \mathrm{G}$ of clean data was obtained from each sample after removing reads of low quality, adaptor contamination and excessively high levels of unknown base N. Approximately $93.09 \%$ of clean reads were aligned to the reference genome (Supplementary Table 2). The average alignment of the gene set was $80.98 \%$, and a total of 23,112 genes were detected.

Differential gene expression was calculated using a Poisson distribution model. Global gene expression profiles under two different water regime conditions are shown on a heatmap by comparing WW-WT/WW-not, and DS-WT/DSnot (Figures 3A,B). The most highly differential expressed genes were visualized using volcano plot. The $\log 2$ values of WW-WT/WW-not, DS-WT/DS-not, DS-WT/WW-WT, and DS-not/WW-not were plotted against - $\log 10$ (Figures 3C-F). Graphical representation of upregulated and downregulated genes is shown in Figure 3G. With the WW-WT/WW-not treatments, a total of 1,578 DEGs, including 916 upregulated and 662 downregulated genes, were identified. Comparatively, with DS-WT/DS-not treatments, a total 1,050 DEGs, including 642 upregulated and 408 downregulated genes, were identified.

\section{DEGs Responsive to ABA and Drought}

The differentially expressed genes (DEGs) were assigned various functions using GO terms. In both DS-WT and DSnot, the most enriched GO terms were ABA and defense responses (Supplementary Figure 3B). Under drought stress conditions, 26 upregulated and 14 downregulated DEGs were related to ABA signaling, whereas 20 upregulated and 

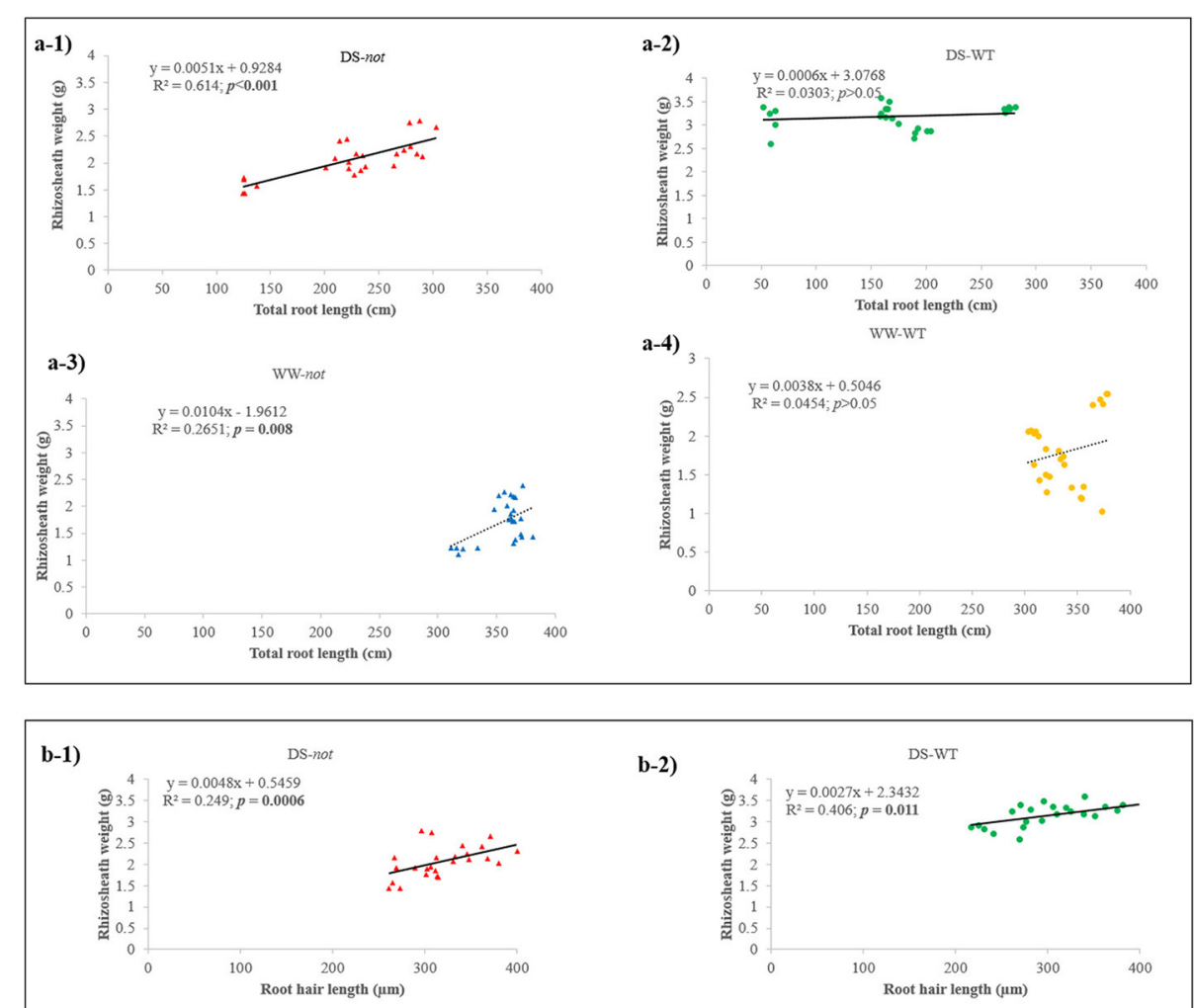

b-2)

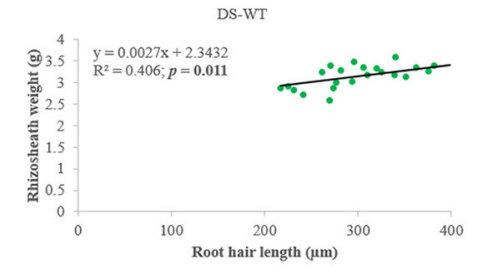

b-3)

b-4)
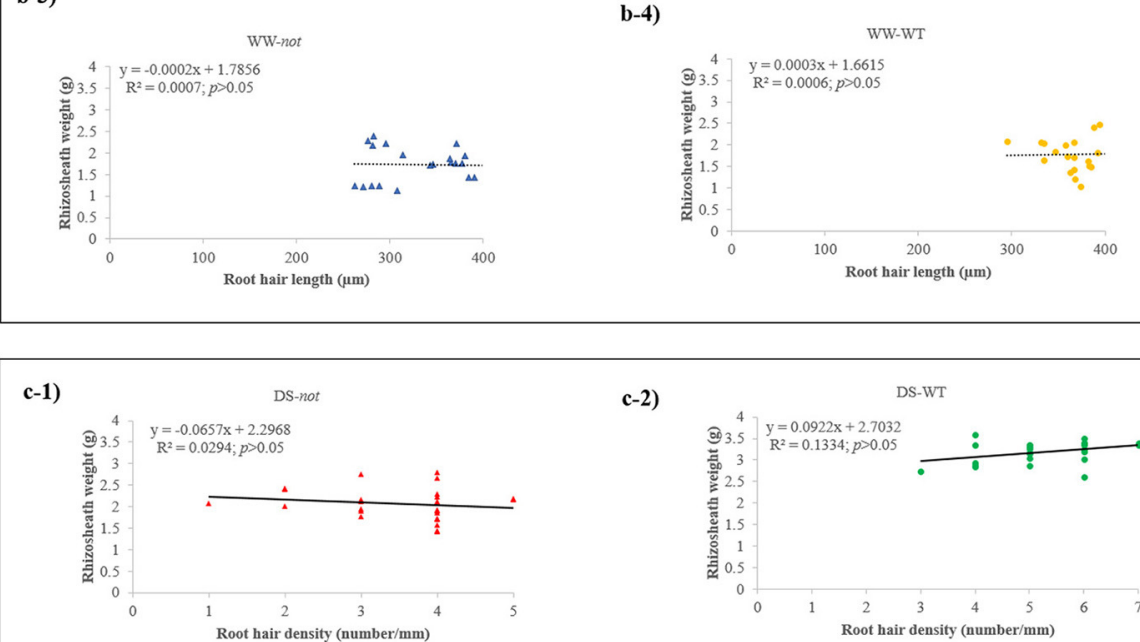

c-2)

c-3)

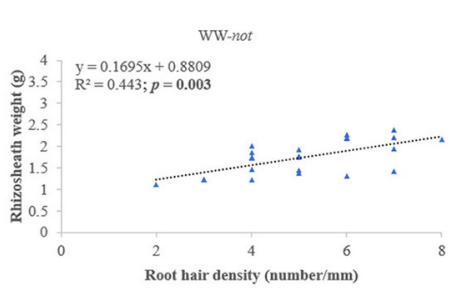

c-4)
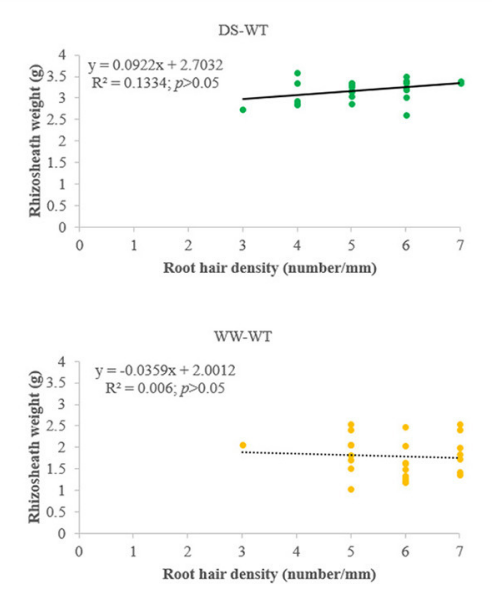

FIGURE 2 | Rhizosheath weight plotted against total root length (a1-a4), root hair length (b1-b4), and root hair density (c1-c4). Each point represents an individual plant, and linear correlations of WT and not plants were fitted where significant $(p \leq 0.05) P$-values are shown in bold for plants grown under drying stress (solid lines) and WW (dashed lines) conditions, with $P$-values and $r^{2}$ reported. WT (orange circles) and not (blue triangles) plants grown under WW conditions; and WT (green circles) and not plants (red triangles) grown under drying stress conditions are indicated. Regression lines are fitted to all panels, with $r^{2}$ and $P$-values indicated. 

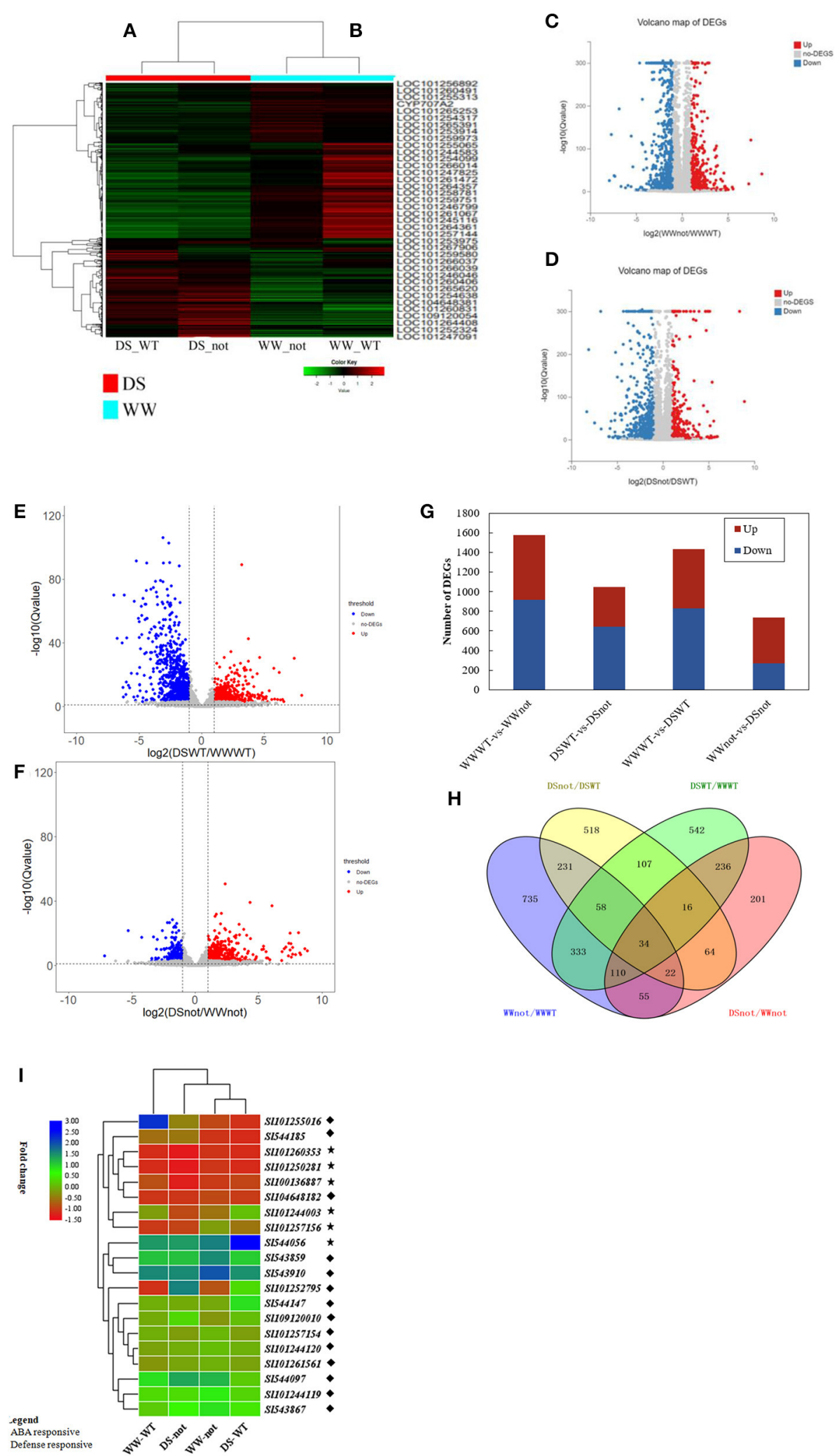

FIGURE 3 | Transcriptional variation in differentially expressed genes of tomato wild-type (WT) and mutant (not) responsive to drying stress and heatmap of DEGs compared under DS-WT and DS-not (A); WW-WT and WW-not (B), based on the expression levels (RPKM+1). Genes in red and green represent highly and lowly 
FIGURE 3 | expressed genes, respectively. (C-F) Volcano plots of the WW-WTMW-not, DS-WT/DS-not, DS-WTMW-WT, and DS-not/WW-not groups. The X-axis represents the fold change in the difference after conversion to log2, and the Y-axis represents the significance value after conversion to -log10. Red represents upregulated DEGs, blue shows downregulated DEGs, while gray represents non-DEGs; (G) graph showing number of up-and downregulated genes; X-axis represents the alignment scheme of DEGs for each group, and the Y-axis represents the corresponding number of DEGs. Red represents the number of upregulated DEGs, and blue represents the number of downregulated DEGs; $\mathbf{( H )}$ Venn diagram showing the number of DEGS with WW and DS treatments (statistically significant $\geq 2$-fold, $p$-value $<0.05$ ); each circle represents a group of gene sets, and the overlapping region represents common DEGs between different treatments; (l) Heatmap of DEGs responsive to ABA and defense, at $p$-value $<0.001$ and log2 (fold change) $>1$.

24 downregulated DEGs were found for defense responses (Figure 3I, Supplementary Datasets 1, 2). Several water deprivation-responsive genes, such as APETALA2-like ethylene responsive transcription factor/AP2-like ERF TF (At1g16060), and dehydrins, such as TAS 14 peptide (LOC_544056), were upregulated by the ABA signaling pathway. In addition, ABA 8'-hydroxylase (LOC_100136887), which encodes an enzyme responsible for ABA catabolism, was also upregulated by ABA. On the other hand, drought upregulated several defenserelated genes, such as lysine $\mathrm{M}$ domain receptor-like kinase (LOC_101260353), pathogenesis-related proteins, protein TIFY 5A-like (LOC_101255016), and tyrosine- and lysinerich proteins, but downregulated protein phosphatase $2 \mathrm{C}$ (Supplementary Datasets 1, 2).

\section{Gene Ontology Functional Analysis of DEGs}

To identify the biological functions, the differentially expressed genes (DEGs) were used to perform gene ontology (GO) analysis based on sequence homologies. Comparing the WW-WT/WWnot group, the four main GO categories in the biological process were cellular processes, metabolic processes, response to stimulus, and biological regulation. In the cellular component, the four main GO terms were cell, cell part, organelle, and membrane; while in the molecular function, binding, catalytic activity, transcription regulator activity, and transporter activity were the four main GO categories (Supplementary Figure 2A). Comparing the DS-WT/DS-not groups, similar GO terms in biological processes, cellular components, and molecular functions were identified (Supplementary Figure 2B).

To further validate the number of genes involved in the GO enrichment analysis, a bubble chart graph was designed. Rich ratio was calculated as follows: rich ratio $=$ term candidate gene number/term gene number. In the WW-WT/WW-not group, the two main gene sets of GO terms in the biological process were 72 genes in defense response and 55 genes responsive to abscisic acid; in cellular component were 313 genes in integral membrane protein and 189 genes in the plasma membrane; and in molecular function were 161 genes in DNA-binding transcription activity and 98 genes in sequence-specific DNA binding (Supplementary Figure 3A). On the other hand, in the DS-WT/DS-not group, the main gene sets of GO terms in the biological process were 44 genes in defense response and 40 genes responsive to abscisic acid; in cellular component were 229 genes in integral membrane protein and 119 genes in plasma membrane; and in molecular function were 38 genes in DNAbinding transcription activity and 98 genes involved in heme binding (Supplementary Figure 3B).

\section{KEGG Enrichment Analysis of DEGs of Tomato Wild-Type and Mutant Not Under Control and Drought Stress Conditions}

To further study the biological pathways of differentially expressed genes (DEGs) triggered by water stress, the DEGs were annotated by blast analysis against the KEGG database. Genes that were up- or downregulated with WW and DS treatments in both WW and mutant tomatoes were subjected to KEGG enrichment analysis (Supplementary Figure 4). A total of 1,578 DEGs identified above were significantly enriched in 44 KEGG pathways in the WW-WT/WW-not and DS-WT/DS-not group comparison. The three major enriched gene KEGG pathways were "Signal transduction," "Global and overview maps," and "Immune system" in both groups.

\section{Root Transcriptional Expression of ABA- and Drought-Related Genes}

RNA-seq results revealed multiple genes involved in ABA and drought signaling pathways. Of these, six candidate ABAand drought-responsive genes that were highly differentially expressed in the two genotypes under WW and drying stress conditions were selected for confirmation by RT-qPCR. They were involved in different pathways of plant defense, namely, the pathogenesis-related protein 4 (PR 4) and protein TIFY 5A-like, and ABA signaling pathways, such as LysM domain receptor-like kinase (Lyk14), APETALA2-like ethyleneresponsive transcription factor (AP2-like ER TF), abscisic acid 8 -hydroxylase, protein phosphatase 2C (PP 2C), and the TAS 14 peptide.

Root gene expression of the pathogenesis-related protein 4 (PR 4) was low under well-watered conditions and in droughted WT plants, but increased more than 4-fold in droughted not plants (Figure 4A). While there were no genotypic differences in root expression profiles of AP2like ER TF under WW conditions, drying stress increased its expression by 5.5-fold in WT roots but had no effect on not roots (Figure 4B). While gene expression of protein phosphatase 2C (PP 2C) was similar in both genotypes under well-watered conditions, drying stress significantly increased its expression by 1.9 -fold in WT roots with an attenuated response in not roots (Figure 4C). Drying stress treatment increased the gene expression of ABA $8^{\prime}$-hydroxylase in WT roots by almost 1.7 -fold, but had no significant effect on not roots (Figure 4D). Taken together, drought-induced ABA accumulation upregulated ABA-related and defense genes, which may have modulated root traits favoring soil adherence to the roots. 

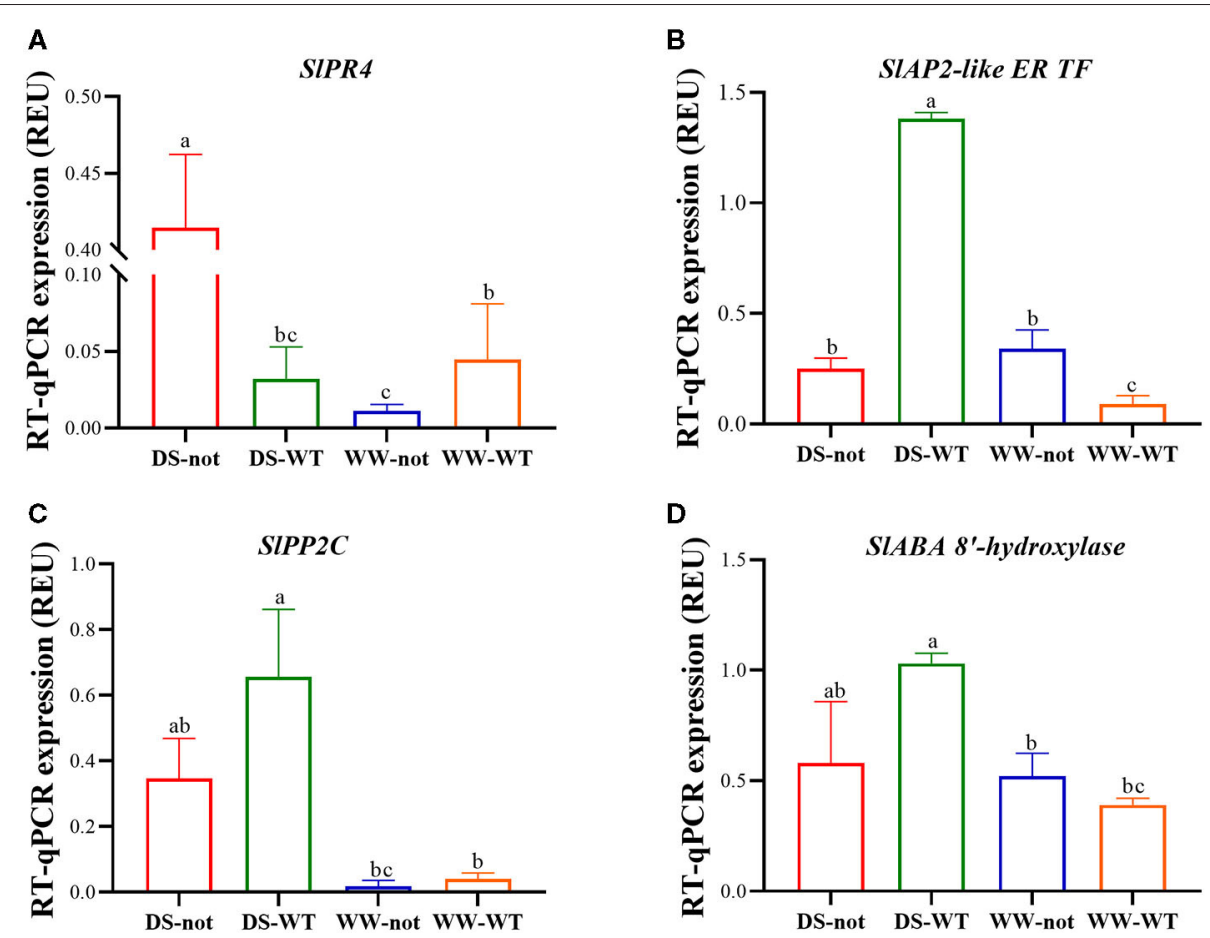

FIGURE 4 | Root expression of genes encoding the Solanum lycopersicum (SI) pathogenesis-related protein 4 (SIPR 4) (A) and APETALA2-like ethylene-responsive transcription factor (SIAP2-like ER TF) (B), protein phosphatase 2C (SIPP 2C) (C), abscisic acid 8'-hydroxylase (SIABA 8'-hydroxylase) (D) based on RT-qPCR data. Columns represent the relative expression levels of genes with different treatments. Bars on top of the columns represent the mean \pm standard error (s.e.) of three biological replicates. The ubiquitin gene was used as an internal control to normalize the expression levels. Significantly different $(p \leq 0.05)$ expression levels with different treatments are indicated with different letters.

\section{DISCUSSION}

ABA-mediated processes help plants adapt to drought by modifying root system architecture, physiological responses, and expression of stress-responsive genes (Cutler et al., 2010). Nonetheless, the role of $\mathrm{ABA}$ in mediating drought-induced rhizosheath formation in crop species is still unclear (Zhang et al., 2020). Comparing the drought stress response of WT and ABA-deficient tomato plants revealed that ABA accumulation, to some extent, inhibited root elongation in dry sand, but stimulated rhizosheath formation, in part by attenuating decreases in root hair density and possibly by modifying stress-responsive gene expression. While root hairs seem important in physically enmeshing soil particles (Koebernick et al., 2017; De Baets et al., 2020), the greater rhizosheath weight of the WT plants per unit of root hair length (Figure 2B) suggests that ABA mediates changes in exudate chemistry that determine rhizosheath formation as the sand dries.

Whereas previous studies demonstrated root growth (volume, surface area, and length) of notabilis was substantially less than that of WT plants when grown in loamy sand (Tracy et al., 2015), here genotypic effects on root length seemed to depend on soil water status. While there were no genotypic differences in root length under WW conditions, the not plants had $23 \%$ longer root length than WT plants when grown under drying stress conditions (Figure 1A), contrary to experiments showing that $\mathrm{ABA}$ is required to maintain primary root elongation of maize plants under drying stress conditions (Spollen et al., 2000) and lateral root development of tomato (Tracy et al., 2015). Nevertheless, the not plants grown in vitro had more and longer lateral roots than the WT plants (Belimov et al., 2014), suggesting that in some substrates, ABA may actually inhibit root growth. Under drying stress condition, roots of the not plants did not accumulate ABA (Figure 1F) in contrast to WT plants, suggesting that drought-induced root $\mathrm{ABA}$ accumulation restricted the root length of the WT plants. Indeed, high concentrations $(>1 \mu \mathrm{M})$ of exogenous ABA can inhibit root growth (Rowe et al., 2016), thus a certain threshold of ABA accumulation is required for root growth beyond which growth is arrested.

In contrast, drying stress promoted the rhizosheath formation of both tomato genotypes as in other crop species (Watt et al., 1994; Albalasmeh and Ghezzehei, 2014). The WT plants having greater rhizosheath than the not plants (Figure 1B) indicates that $\mathrm{ABA}$ is needed for maximal rhizosheath development as the sand dries. Both genotypes had similar rhizosheath development under WW conditions consistent with their similar root $\mathrm{ABA}$ concentration under these conditions. Likewise, the ABA-deficient barley mutant $A z 34$ had restricted rhizosheath 
development independent of soil water status, associated with its lower root ABA concentration (Zhang et al., 2021). Nevertheless, the mechanisms by which root ABA accumulation promote rhizosheath formation require further investigation.

Growth of root hairs is highly plastic and is regulated by a wide range of both endogenous and environmental inputs (Bustos et al., 2010; Zhu et al., 2010; Chandrika et al., 2013). Root hairs greatly increase root-soil contact to facilitate nutrient and water absorption (Haling et al., 2013), implying that the length and/or density of root hairs formed helps plants adapt to stressful conditions. While rhizosheath weight was directly correlated with root hair length in wheat (Delhaize et al., 2012), these traits were weakly associated in an array of barley genotypes (George et al., 2014), and no association was observed in diverse chickpea genotypes (Pang et al., 2017). Whereas drying stress increased root hair length of cotton (Xiao et al., 2020) and orange (Zhang et al., 2019), root hair length of both tomato genotypes decreased under drying stress conditions (Figure 1D, Table 1). Nevertheless, the roots of the WT plants held more sand per unit of root hair length as the sand dried (Figure 2B), which may help acquire soil moisture by increasing root-soil contact in sandy soils (North and Nobel, 1997; Smith et al., 2011).

Although the WT plants had longer and more numerous root hairs than not under WW conditions (Figures 1D,E), this did not affect rhizosheath development. Drying the sand substantially decreased the root hair density of tomato (Figure 1E), in contrast to the increase in other species (Liu et al., 2019; Zhang et al., 2019). Under drying stress conditions, the root hair density of the WT plants was 33\% higher than that of the not plants, consistent with the genotypic differences in rhizosheath weight. While this ABA-mediated attenuation of the decline in root hair density with sand drying is associated with greater rhizosheath development of the WT plants, it is interesting that rhizosheath weight was highly correlated with root hair length in both genotypes under drying stress conditions (Figure 2B), with the WT plants binding 1.5-fold more sand at the same root hair length. This may also imply differences in root hair chemistry affecting binding of sand particles (Watt et al., 1994; Haling et al., 2010, 2014; George et al., 2014; Pang et al., 2017).

Transcriptome analysis revealed that drought-related and ABA signaling pathway genes were differentially regulated under drought. TFs serve as master regulators of cellular processes by regulating downstream genes related to drought tolerance (Gahlaut et al., 2016; Joshi et al., 2016), and AP2/ERFs can regulate drought stress responses in $S$. lycopersicum (Wu et al., 2008). Drying stress upregulated root SIERF5 expression, while overexpressing SIERF5 increased drought stress tolerance by increasing leaf relative water content (Pan et al., 2012). Upregulation ofAP2/ERF transcription factor genes in the roots in dry sand was ABA-dependent (Figure 4B) and may be important in ABA-mediated rhizosheath development, by affecting as yet unidentified mechanisms.

Although SlERF5 overexpression in tomato stimulated foliar expression of pathogenesis-related (PR) defense genes (Li et al., 2011), the root PR4 gene expression under drying stress conditions showed an opposite response to AP2/ERF transcription factor gene expression (cf. Figures 4A,B). Upregulation of the PR4 expression in the not roots in drying stress (Figure 4A) was independent of both ABA and AP2/ERF transcription factor gene expressions, which increased only in WT roots under drying stress conditions. In contrast, both exogenous ABA and drought upregulated the OsPR4 expression in rice leaves (Wang et al., 2011), with relatively low expression levels in the roots compared with above-ground tissues. Notwithstanding possible differential regulation of PR4 genes in rice and tomato, enhanced root PR4 gene expression in not may enhance resistance against biotic stresses, at least under drying stress conditions. In support, not was less susceptible to root-knot nematode infection, associated with its higher constitutive and nematode-induced expression of the defenserelated gene plant defense factor (PDF) and the proteinase inhibitors PI-1 and PI-2 (Xu et al., 2019). Whether PR4 is associated with rhizosheath formation in drying stress conditions requires rhizosheath measurements in genotypes under- or overexpressing PR4 (Wang et al., 2011).

In plants, reversible protein phosphorylation mediated by protein phosphatases and kinases is an important adaptive cellular response maintaining phospho-regulation under normal and stressful growth conditions. Protein phosphatases, especially Clade A of PP 2C, have been implicated in Arabidopsis and rice signaling pathways triggered by stress such as drought (Singh et al., 2010; He et al., 2019). Drought-induced upregulation of the root PP 2C gene expression in the WT roots was consistent with increased root $\mathrm{ABA}$ concentration (cf. Figures 1F, 4C), whereas high variability of the root $\mathrm{PP} 2 \mathrm{C}$ gene expression in the not roots was inconsistent with the lack of root ABA accumulation. Taken together, the ABA-dependent expression of PP $2 \mathrm{C}$ genes in roots may play important roles in regulating root growth response to drying stress.

Although genotypic and drought-induced differences in root ABA concentrations were consistent with the expected phenotypes based on knowledge of NCED gene expression (Thompson et al., 2000), stress-induced ABA accumulation can also be modulated by ABA conjugation or oxidation, especially ABA 8'-hydroxylation catalyzed by CYP707A proteins (Kushiro et al., 2004; Saito et al., 2004). Although drying stress treatment stimulated the expression of an ABA $8^{\prime}$-hydroxylase (CYP707A1) gene in the WT roots (Figure 4D), implying that CYP707A1 might be involved in ABA catabolism in these tomato roots, root ABA concentration still increased in association with root length inhibition compared with the not plants. Thus, ABA biosynthesis was greater than ABA metabolism in the WT roots, although the import of ABA from the shoot (McAdam et al., 2016) might provide an additional source of ABA.

In conclusion, gene expression responses to drying stress induced root $\mathrm{ABA}$ accumulation and expression of associated signaling genes in the WT plants. Although these responses diminished root exploration of the dry sand compared with the not plants, they greatly promoted rhizosheath formation. This ABA-dependent stimulation of rhizosheath development under drying stress conditions was independent of root hair length and 
density, suggesting that future studies should focus on the effects of root ABA status on exudate chemistry.

\section{DATA AVAILABILITY STATEMENT}

The datasets presented in this study can be found in online repositories. The names of the repository/repositories and accession number(s) can be found at: PRJNA731295, https:// www.ncbi.nlm.nih.gov/bioproject/PRJNA731295.

\section{AUTHOR CONTRIBUTIONS}

XW and ID conceived the study and supervised the whole research. JK performed most of the experiments, analyzed the data, and wrote the manuscript. MA, ZQ, XW, and RY reviewed the study. ID critically reviewed and revised the study. All authors contributed to the article and approved the submitted version.

\section{FUNDING}

This study was supported by grants from the National Key R\&D Program of China (2017YFE0118100, 2018YFD02003025, and 2018YFD1000800), National Natural Science Foundation of China (Nos. 31872169, 31722048, and 31761130073), and Newton Advanced Fellowship (NSFC-RS: NA160430).

\section{REFERENCES}

Albalasmeh, A. A., and Ghezzehei, T. A. (2014). Interplay between soil drying and root exudation in rhizosheath development. Plant Soil. 374:739-751. doi: 10.1007/s11104-013-1910-y

Anjum, S. A., Ashraf, U., Zohaib, A., Tanveer, M., Naeem, M., Ali, I., et al. (2017). Growth and development responses of crop plants under drought stress: a review. Zemdirbyste 104, 267-276. doi: 10.13080/z-a.2017.104.034

Battisti, D. S., and Naylor, R. L. (2009). Historical warnings of future food insecurity with unprecedented seasonal heat. Science 323, 240-244. doi: 10.1126/science. 1164363

Belimov, A. A., Dodd, I. C., Safronova, V. I., Dumova, V. A., Shaposhnikov, A. I., Ladatko, A. G., et al. (2014). Abscisic acid metabolizing rhizobacteria decrease ABA concentrations in planta and alter plant growth. Plant Physiol. Biochem. 74, 84-91. doi: 10.1016/j.plaphy.2013.10.032

Benjamini, Y., and Hochberg, Y. (1995). Controlling the false discovery rate: a practical and powerful approach to multiple testing. J. R. Stat. Soc. Ser. B 57, 289-300. doi: 10.1111/j.2517-6161.1995.tb02031.x

Bustos, R., Castrillo, G., Linhares, F., Puga, M. I., Rubio, V., Pérez-Pérez, J., et al. (2010). A central regulatory system largely controls transcriptional activation and repression responses to phosphate starvation in Arabidopsis. PLoS Genet. 6:e1001102. doi: 10.1371/journal.pgen.1001102

Carminati, A., Benard, P., Ahmed, M. A., and Zarebanadkouki, M. (2017). Liquid bridges at the root-soil interface. Plant Soil 417, 1-15. doi: 10.1007/s11104-017-3227-8

Chandrika, N. N. P., Sundaravelpandian, K., Yu, S. M., and Schmidt, W. (2013). ALFIN-LIKE 6 is involved in root hair elongation during phosphate deficiency in Arabidopsis. New Phytol. 198, 709-720. doi: 10.1111/nph.12194

Chen, C.-W., Yang, Y.-W., Lur, H. S., Tsai, Y.-G., and Chang, M.-C. (2006). A novel function of abscisic acid in the regulation of rice (Oryza sativa L.) root growth and development. Plant Cell Physiol. 47, 1-13. doi: 10.1093/pcp/pci216

Cutler, S. R., Rodriguez, P. L., Finkelstein, R. R., and Abrams, S. R. (2010). Abscisic acid: emergence of a core signaling network. Annu.

\section{SUPPLEMENTARY MATERIAL}

The Supplementary Material for this article can be found online at: https://www.frontiersin.org/articles/10.3389/fpls.2021. 658787/full\#supplementary-material

Supplementary Figure 1 | Rhizosheath formation of (A) notabilis and (B) wild-type plants growing under drying stress conditions; and (C) notabilis and (D) wild-type plants growing under well-watered conditions, (E) root hair analysis as determined using the Image $\mathrm{J}$ software, and $(\mathbf{F})$ root length as determined by the WinRhizo program.

Supplementary Figure 2 | GO enrichment analysis of the DEGs in two different group comparisons.

Supplementary Figure 3 | GO enrichment analysis of DEGs represented by bubble chart.

Supplementary Figure 4 | KEGG enrichment analysis of the two-group comparison.

Supplementary Dataset 1 | Differentially expressed genes (DEGs) related to ABA response (Excel file separately attached).

Supplementary Dataset 2 | Differentially expressed genes (DEGs) related to defense response (Excel file separately attached).

Supplementary Table 1 | Primers used for RT-qPCR expression analysis.

Supplementary Table 2 | Summary of read numbers and alignment obtained from RNA sequence of drought-stressed and control samples.

Supplementary Table 3 | Fold changes in gene expression in rhizosheath-bound roots under drying stress compared to well-watered conditions in two tomato genotypes (Lukullus and notabilis) determined by RNA-seq and RT-qPCR.

Rev. Plant Biol. 61, 651-679. doi: 10.1146/annurev-arplant-042809-1 12122

Czechowski, T., Stitt, M., Altmann, T., Udvardi, M. K., and Scheible, W.R. (2005). Genome-wide identification and testing of superior reference genes for transcript normalization in Arabidopsis. Plant Physiol. 139, 5-17. doi: $10.1104 /$ pp.105.063743

Davies, P. J. (2010). “The plant hormones: their nature, occurrence, and functions," in Plant Hormones (Springer), 1-15. doi: 10.1007/978-1-4020-2686-7_1

De Baets, S. T., Denbigh, D. G., Smyth, K. M., Eldridge, B. M., Weldon, L., et al. (2020). Micro-scale interactions between Arabidopsis root hairs and soil particles influence soil erosion. Commun. Biol. 3, 1-11. doi: 10.1038/s42003-020-0886-4

Delhaize, E., James, R. A., and Ryan, P. R. (2012). Aluminium tolerance of root hairs underlies genotypic differences in rhizosheath size of wheat (Triticum aestivum) grown on acid soil. New Phytol. 195, 609-619. doi: 10.1111/j.1469-8137.2012.04183.x

Delhaize, E., Rathjen, T. M., and Cavanagh, C. R. (2015). The genetics of rhizosheath size in a multiparent mapping population of wheat. J. Exp. Bot. 66, 4527-4536. doi: 10.1093/jxb/erv223

Dodd, I. C., and Diatloff, E. (2016). Enhanced root growth of the brb (bald root barley) mutant in drying soil allows similar shoot physiological responses to soil water deficit as wild-type plants. Funct. Plant Biol. 43, 199-206. doi: 10.1071/FP15303

Gahlaut, V., Jaiswal, V., Kumar, A., and Gupta, P. K. (2016). Transcription factors involved in drought tolerance and their possible role in developing drought tolerant cultivars with emphasis on wheat (Triticum aestivum L.). Theor. Appl. Genet. 129, 2019-2042. doi: 10.1007/s00122-016-2794-z

Gamuyao, R., Chin, J. H., Pariasca-Tanaka, J., Pesaresi, P., Catausan, S., Dalid, C., et al. (2012). The protein kinase Pstoll from traditional rice confers tolerance of phosphorus deficiency. Nature 488, 535-539. doi: 10.1038/nature 11346

George, T. S., Brown, L. K., Ramsay, L., White, P. J., Newton, A. C., Bengough, A. G., et al. (2014). Understanding the genetic control and physiological traits 
associated with rhizosheath production by barley (Hordeum vulgare). New Phytol. 203, 195-205. doi: 10.1111/nph.12786

Giuliani, S., Sanguineti, M. C., Tuberosa, R., Bellotti, M., Salvi, S., and Landi, P. (2005). Root-ABA1, a major constitutive QTL, affects maize root architecture and leaf $\mathrm{ABA}$ concentration at different water regimes. J. Exp. Bot. 56, 3061-3070. doi: 10.1093/jxb/eri303

Haling, R. E., Brown, L. K., Bengough, A. G., Valentine, T. A., White, P. J., Young, I. M., et al. (2014). Root hair length and rhizosheath mass depend on soil porosity, strength and water content in barley genotypes. Planta 239, 643-651. doi: 10.1007/s00425-013-2002-1

Haling, R. E., Brown, L. K., Bengough, A. G., Young, I. M., Hallett, P. D., White, P. J., et al. (2013). Root hairs improve root penetration, root-soil contact, and phosphorus acquisition in soils of different strength. J. Exp. Bot. 64, 3711-3721. doi: $10.1093 /$ jxb/ert200

Haling, R. E., Richardson, A. E., Culvenor, R. A., Lambers, H., and Simpson, R. J. (2010). Root morphology, root-hair development and rhizosheath formation on perennial grass seedlings is influenced by soil acidity. Plant Soil 335, 457-468. doi: 10.1007/s11104-010-0433-z

Hanna, A. L., Youssef, H. H., Amer, W. M., Monib, M., Fayez, M., and Hegazi, N. A. (2013). Diversity of bacteria nesting the plant cover of north Sinai deserts, Egypt. J. Adv. Res. 4, 13-26. doi: 10.1016/j.jare.2011.11.003

He, Z., Wu, J., Sun, X., and Dai, M. (2019). The maize clade A PP2C phosphatases play critical roles in multiple abiotic stress responses. Int. J. Mol. Sci. 20:3573. doi: 10.3390/ijms20143573

Humbeck, K., Kloppstech, K., and Krupinska, K. (1994). Expression of early light-inducible proteins in flag leaves of field-grown barley. Plant Physiol. 105, 1217-1222. doi: 10.1104/pp.105.4.1217

Hussain, M., Farooq, S., Hasan, W., Ul-Allah, S., Tanveer, M., Farooq, M., et al. (2018). Drought stress in sunflower: physiological effects and its management through breeding and agronomic alternatives. Agric. Water Manage. 201, 152-166. doi: 10.1016/j.agwat.2018.01.028

Janiak, A., Kwaśniewski, M., and Szarejko, I. (2016). Gene expression regulation in roots under drought. J. Exp. Bot. 67, 1003-1014. doi: 10.1093/jxb/erv512

Jeong, J. S., Kim, Y. S. M. C., Redillas, F. R., Jang, G., Jung, H., et al. (2013). OsNAC5 overexpression enlarges root diameter in rice plants leading to enhanced drought tolerance and increased grain yield in the field. Plant Biotechnol. J. 11, 101-114. doi: 10.1111/pbi.12011

Joshi, R., Wani, S. H., Singh, B., Bohra, A., Dar, Z. A., Lone, A. A., et al. (2016). Transcription factors and plants response to drought stress: current understanding and future directions. Front. Plant Sci. 7:1029. doi: 10.3389/fpls.2016.01029

Koebernick, N., Daly, K. R., Keyes, S. D., George, T. S., Brown, L. K., Raffan, A., et al. (2017). High-resolution synchrotron imaging shows that root hairs influence rhizosphere soil structure formation. New Phytol. 216, 124-135. doi: $10.1111 /$ nph.14705

Kushiro, T., Okamoto, M., Nakabayashi, K., Yamagishi, K., Kitamura, S., Asami, T., et al. (2004). The Arabidopsis cytochrome P450 CYP707A encodes ABA 8'-hydroxylases: key enzymes in ABA catabolism. EMBO J. 23, 1647-1656. doi: $10.1038 /$ sj.emboj.7600121

Kwasniewski, M., Daszkowska-Golec, A., Janiak, A., Chwialkowska, K., Nowakowska, U., Sablok, G., et al. (2016). Transcriptome analysis reveals the role of the root hairs as environmental sensors to maintain plant functions under water-deficiency conditions. J. Exp. Bot. 67, 1079-1094. doi: $10.1093 / j x b / e r v 498$

Li, B., and Dewey, C. N. (2011). RSEM: accurate transcript quantification from RNA-Seq data with or without a reference genome. BMC Bioinformatics 12:323. doi: 10.1186/1471-2105-12-323

Li, C.-W., Su, R. C., Cheng, C. P., You, S. J., Hsieh, T. H., Chao, T.-C., et al. (2011). Tomato RAV transcription factor is a pivotal modulator involved in the AP2/EREBP-mediated defense pathway. Plant Physiol. 156, 213-227. doi: 10.1104/pp.111.174268

Li, J., Zhu, S., Song, X., Shen, Y., Chen, H., Yu, J., et al. (2006). A rice glutamate receptor-like gene is critical for the division and survival of individual cells in the root apical meristem. Plant Cell 18, 340-349. doi: 10.1105/tpc.105.037713

Liu, S., Wang, X., Wang, H., Xin, H., Yang, X., Yan, J., Li, J., et al. (2013). Genomewide analysis of $Z \mathrm{mDREB}$ genes and their association with natural variation in drought tolerance at seedling stage of Zea mays L. PLoS Genet. 9:e1003790. doi: 10.1371 /journal.pgen. 1003790
Liu, T. Y., Ye, N., Song, T., Cao, Y., Gao, B., Zhang, D., et al. (2019). Rhizosheath formation and involvement in foxtail millet (Setaria italica) root growth under drought stress. J. Integr. Plant Biol. 61, 449-462. doi: 10.1111/jipb.12716

Livak, K. J., and Schmittgen, T. D. (2001). Analysis of relative gene expression data using real-time quantitative PCR and the 2- $\Delta \Delta$ CT method. Methods 25, 402-408. doi: 10.1006/meth.2001.1262

Long, S. P., Marshall-Colon, A., and Zhu, X.-G. (2015). Meeting the global food demand of the future by engineering crop photosynthesis and yield potential. Cell 161, 56-66. doi: 10.1016/j.cell.2015.03.019

Lynch, J. (1995). Root architecture and plant productivity. Plant Physiol. 109:7. doi: 10.1104/pp.109.1.7

Lynch, J. P., Chimungu, J. G., and Brown, K. M. (2014). Root anatomical phenes associated with water acquisition from drying soil: targets for crop improvement. J. Exp. Bot. 65, 6155-6166. doi: 10.1093/jxb/eru162

Mach, K. J., Kraan, C. M., Adger, W. N., Buhaug, H., Burke, M., Fearon, J. D., et al. (2019). Climate as a risk factor for armed conflict. Nature 571, 193-197. doi: 10.1038/s41586-019-1300-6

Mahalingam, R. (ed.). (2015). "Consideration of combined stress: a crucial paradigm for improving multiple stress tolerance in plants," in Combined Stresses in Plants (Stillwater, OK: Springer), 1-25. doi: 10.1007/978-3-319-07899-1_1

Marin, M., Feeney, D. S., Brown, L. K., Naveed, M., Ruiz, S., Koebernick, N., et al. (2020). Significance of root hairs for plant performance under contrasting field conditions and water deficit. Ann. Bot. doi: 10.1093/aob/mcaa181

Mascher, M., Gundlach, H., Himmelbach, A., Beier, S., Twardziok, S. O., Wicker, T., et al. (2017). A chromosome conformation capture ordered sequence of the barley genome. Nature 544, 427-433. doi: 10.1038/nature22043

McAdam, S. A. M. (2015). Physicochemical quantification of abscisic acid levels in plant tissues with an added internal standard by ultra-performance liquid chromatography. Bio-Protocol 5, 1-13. doi: 10.21769/BioProtoc.1599

McAdam, S. A. M., Brodribb, T. J., and Ross, J. J. (2016). Shoot-derived abscisic acid promotes root growth. Plant Cell Environ. 39, 652-659. doi: 10.1111/pce.12669

McCully, M. E. (1999). Roots in soil: unearthing the complexities of roots and their rhizospheres. Annu. Rev. Plant Biol. 50, 695-718. doi: 10.1146/annurev.arplant.50.1.695

Nestler, J., Keyes, S. D., and Wissuwa, M. (2016). Root hair formation in rice (Oryza sativa L.) differs between root types and is altered in artificial growth conditions. J. Exp. Bot. 67, 3699-3708. doi: 10.1093/jxb/erw115

Ning, P., Liao, C., Li, S., Yu, P., Zhang, Y., Li, X., et al. (2012). Maize cob plus husks mimics the grain sink to stimulate nutrient uptake by roots. Field Crops Res. 130, 38-45. doi: 10.1016/j.fcr.2012.02.010

North, G. B., and Nobel, P. S. (1997). Root-soil contact for the desert succulent Agave deserti in wet and drying soil. New Phytol. 135, 21-29. doi: 10.1046/j.1469-8137.1997.00620.x

Pan, Y., Seymour, G. B., Lu, C., Hu, Z., Chen, X., and Chen, G. (2012). An ethylene response factor (ERF5) promoting adaptation to drought and salt tolerance in tomato. Plant Cell Rep. 31, 349-360. doi: 10.1007/s00299-011-1170-3

Pang, J., Ryan, M. H. K., Siddique, H. M., and Simpson, R. J. (2017). Unwrapping the rhizosheath. Plant Soil 418, 129-139. doi: 10.1007/s11104-0173358-y

Pereg, L., and McMillan, M. (2015). Scoping the potential uses of beneficial microorganisms for increasing productivity in cotton cropping systems. Soil Biol. Biochem. 80, 349-358. doi: 10.1016/j.soilbio.2014.10.020

Peterson, R. L., and Farquhar, M. L. (1996). Root hairs: specialized tubular cells extending root surfaces. Botan. Rev. 62, 1-40. doi: 10.1007/BF028 68919

Recchia, G. H. D., Caldas, G. G. A., Beraldo, L. A., Da Silva, M. J., et al. (2013). Transcriptional analysis of drought-induced genes in the roots of a tolerant genotype of the common bean (Phaseolus vulgaris L.). Int. J. Mol. Sci. 14, 7155-7179. doi: 10.3390/ijms14047155

Richardson, A. E., Barea, J. M., McNeill, A. M., and Prigent-Combaret, C. (2009). Acquisition of phosphorus and nitrogen in the rhizosphere and plant growth promotion by microorganisms. Plant Soil 321, 305-339. doi: 10.1007/s11104-009-9895-2

Rowe, J. H., Topping, J. F., Liu, J., and Lindsey, K. (2016). Abscisic acid regulates root growth under osmotic stress conditions via an interacting hormonal network with cytokinin, ethylene and auxin. New Phytol. 211, 225-239. doi: $10.1111 /$ nph.13882 
Rymen, B., Kawamura, A., Schäfer, S., Breuer, C., Iwase, A., Shibata, M., et al. (2017). ABA suppresses root hair growth via the OBP4 transcriptional regulator. Plant Physiol. 173, 1750-1762. doi: 10.1104/pp.16.01945

Saab, I. N., Sharp, R. E., Pritchard, J., and Voetberg, G. S. (1990). Increased endogenous abscisic acid maintains primary root growth and inhibits shoot growth of maize seedlings at low water potentials. Plant Physiol. 93, 1329-1336. doi: $10.1104 /$ pp.93.4.1329

Saito, S., Hirai, N., Matsumoto, C., Ohigashi, H., Ohta, D., Sakata, K., et al. (2004). Arabidopsis CYP707As encode (+)-abscisic acid 8' -hydroxylase, a key enzyme in the oxidative catabolism of abscisic acid. Plant Physiol. 134, 1439-1449. doi: 10.1104/pp.103.037614

Schachtman, D. P., and Goodger, J. Q. D. (2008). Chemical root to shoot signaling under drought. Trends Plant Sci. 13, 281-287. doi: 10.1016/j.tplants.2008.04.003

Sharipova, G., Veselov, D., Kudoyarova, G., Fricke, W., Dodd, I. C., Katsuhara, M., et al. (2016). Exogenous application of abscisic acid (ABA) increases root and cell hydraulic conductivity and abundance of some aquaporin isoforms in the ABA-deficient barley mutant Az34. Ann. Bot. 118, 777-785. doi: $10.1093 / \mathrm{aob} / \mathrm{mcw} 117$

Singh, A., Giri, J., Kapoor, S., Tyagi, A. K., and Pandey, G. K. (2010). Protein phosphatase complement in rice: genome-wide identification and transcriptional analysis under abiotic stress conditions and reproductive development. BMC Genomics 11:435. doi: 10.1186/1471-2164-11-435

Smith, R. J., Hopper, S. D., and Shane, M. W. (2011). Sand-binding roots in Haemodoraceae: global survey and morphology in a phylogenetic context. Plant Soil 348:453. doi: 10.1007/s11104-011-0874-z

Smith, S., and De Smet, I. (2012). Root system architecture: insights from Arabidopsis and cereal crops. Philos. Trans. R. Soc. Lond. B Biol. Sci. 367, 1441-1452. doi: 10.1098/rstb.2011.0234

Spollen, W. G., LeNoble, M. E., Samuels, T. D., Bernstein, N., and Sharp, R. E. (2000). Abscisic acid accumulation maintains maize primary root elongation at low water potentials by restricting ethylene production. Plant Physiol. 122, 967-976. doi: 10.1104/pp.122.3.967

Thompson, A. J., Jackson, A. C., Parker, R. A., Morpeth, D. R., Burbidge, A., and Taylor, I. B. (2000). Abscisic acid biosynthesis in tomato: regulation of zeaxanthin epoxidase and 9-cis-epoxycarotenoid dioxygenase mRNAs by light/dark cycles, water stress and abscisic acid. Plant Mol. Biol. 42, 833-845. doi: 10.1023/A:1006448428401

Thompson, A. J., Thorne, E. T., Burbidge, A., Jackson, A. C., Sharp, R. E., and Taylor, I. B. (2004). Complementation of notabilis, an abscisic acid-deficient mutant of tomato: importance of sequence context and utility of partial complementation. Plant Cell Environ. 27, 459-471. doi: 10.1111/j.1365-3040.2003.01164.x

Tracy, S. R., Black, C. R., Roberts, J. A., Dodd, I. C., and Mooney, S. J. (2015). Using $\mathrm{X}$-ray computed tomography to explore the role of abscisic acid in moderating the impact of soil compaction on root system architecture. Environ. Exp. Bot. 110, 11-18. doi: 10.1016/j.envexpbot.2014.09.003

Uga, Y., Sugimoto, K., Ogawa, S., Rane, J., Ishitani, M., Hara, N., et al. (2013). Control of root system architecture by DEEPER ROOTING 1 increases rice yield under drought conditions. Nat. Genet. 45:1097-1102. doi: 10.1038/ng.2725

Wang, N., Xiao, B., and Xiong, L. (2011). Identification of a cluster of PR4-like genes involved in stress responses in rice. J. Plant Physiol. 168, 2212-2224. doi: 10.1016/j.jplph.2011.07.013

Wang, T., Li, C., Wu, Z., Jia, Y., Wang, H., Sun, S., et al. (2017). Abscisic acid regulates auxin homeostasis in rice root tips to promote root hair elongation. Front. Plant Sci. 8:1121. doi: 10.3389/fpls.2017.01121

Watt, M., McCully, M. E., and Canny, M. J. (1994). Formation and stabilization of rhizosheaths of Zea mays L.(Effect of soil water content). Plant Physiol. 106, 179-186. doi: 10.1104/pp.106.1.179
White, P. J., George, T. S., Dupuy, L. X., Karley, A. J., Valentine, T. A., Wiesel, L., et al. (2013a). Root traits for infertile soils. Front. Plant Sci. 4:193. doi: 10.3389/fpls.2013.00193

White, P. J., George, T. S., Gregory, P. J., Bengough, A. G., Hallett, P. D., and McKenzie, B. M. (2013b). Matching roots to their environment. Ann. Bot. 112, 207-222. doi: 10.1093/aob/mct123

Wu, L., Zhang, Z., Zhang, H., X.-,Wang, C., and Huang, R. (2008). Transcriptional modulation of ethylene response factor protein JERF3 in the oxidative stress response enhances tolerance of tobacco seedlings to salt, drought, and freezing. Plant Physiol. 148, 1953-1963. doi: 10.1104/pp.108.126813

Xiao, S., Liu, L., Zhang, Y., Sun, H., Zhang, K., Bai, Z., et al. (2020). Fine root and root hair morphology of cotton under drought stress revealed with RhizoPot. J. Agron. Crop Sci. 206, 679-693. doi: 10.1111/jac.12429

Xie, C., Mao, X., Huang, J., Ding, Y., Wu, J., Dong, S., et al. (2011). KOBAS 2.0: a web server for annotation and identification of enriched pathways and diseases. Nucleic Acids Res. 39, W316-W322. doi: 10.1093/nar/ gkr483

Xu, W., Jia, L., Shi, W., Liang, J., Zhou, F., Li, Q., et al. (2013). Abscisic acid accumulation modulates auxin transport in the root tip to enhance proton secretion for maintaining root growth under moderate water stress. New Phytol. 197, 139-150. doi: 10.1111/nph.12004

Xu, X., Fang, P., Zhang, H., Chi, C., Song, L., Xia, X., et al. (2019). Strigolactones positively regulate defense against root-knot nematodes in tomato. J. Exp. Bot. 70, 1325-1337. doi: 10.1093/jxb/ery439

Zhan, A., Schneider, H., and Lynch, J. P. (2015). Reduced lateral root branching density improves drought tolerance in maize. Plant Physiol. 168, 1603-1615. doi: $10.1104 /$ pp.15.00187

Zhang, F., Wang, P., Zou, Y.-N., Wu, Q.-S., and Kuča, K. (2019). Effects of mycorrhizal fungi on root-hair growth and hormone levels of taproot and lateral roots in trifoliate orange under drought stress. Arch. Agron. Soil Sci. 65, 1316-1330. doi: 10.1080/03650340.2018.1563780

Zhang, Y., Du, H., Gui, Y., Xu, F., Liu, J., Zhang, J., et al. (2020). Moderate water stress in rice induces rhizosheath formation associated with abscisic acid and auxin responses. J. Exp. Bot. 71, 2740-2751. doi: 10.1093/jxb/eraa021

Zhang, Y., Xu, F., Ding, Y., Du, H., Zhang, Q., Dang, X., et al. (2021). Abscisic acid mediates barley rhizosheath formation under mild soil drying by promoting root hair growth and auxin response. Plant Cell Environ. 44, 1935-1945. doi: 10.1111 pce. 14036

Zhu, J., Zhang, C., and Lynch, J. P. (2010). The utility of phenotypic plasticity of root hair length for phosphorus acquisition. Funct. Plant Biol. 37, 313-322. doi: 10.1071/FP09197

Conflict of Interest: The authors declare that the research was conducted in the absence of any commercial or financial relationships that could be construed as a potential conflict of interest.

Publisher's Note: All claims expressed in this article are solely those of the authors and do not necessarily represent those of their affiliated organizations, or those of the publisher, the editors and the reviewers. Any product that may be evaluated in this article, or claim that may be made by its manufacturer, is not guaranteed or endorsed by the publisher.

Copyright (C) 2021 Karanja, Aslam, Qian, Yankey, Dodd and Weifeng. This is an open-access article distributed under the terms of the Creative Commons Attribution License (CC BY). The use, distribution or reproduction in other forums is permitted, provided the original author(s) and the copyright owner(s) are credited and that the original publication in this journal is cited, in accordance with accepted academic practice. No use, distribution or reproduction is permitted which does not comply with these terms. 